\title{
Capital humano: uma nova proxy para incluir aspectos qualitativos 1
}

Luciano Nakabashi ${ }^{2}$

Lízia de Figueiredo3

Resumo: Apesar de já existir um razoável consenso de que o papel do capital humano sobre o nível de renda e taxa de crescimento dos países são cruciais, os resultados empíricos não são tão favoráveis a essa idéia. Muitos estudos empíricos não encontram uma relação positiva significativa entre nível ou taxa de crescimento da renda e nível de capital humano. Aparentemente, os dois principais motivos para que isso aconteça é o fato de que a relação entre essas variáveis pode ser mais complexa do que é sugerido por alguns modelos de crescimento endógeno do tipo Lucas (1988) e Uzawa (1965) e pela proxy que geralmente é usada. O objetivo do presente trabalho foca nesse segundo problema através da utilização de uma proxy que incorpore características quantitativas e qualitativas do fator capital humano. O trabalho e a base de dados de Mankiw, Romer e Weil (1992) são utilizados como base de comparação dos resultados.

Palavras-chave: capital humano; resultados empíricos; nova proxy.

\section{Human capital: a new proxy to capture qualitative aspects}

\footnotetext{
Abstract: Despite the fact that there is already a reasonable consensus about the relevance of the human capital factor on income level and growth rate across countries, the empirical results do not give much support to this idea. Many empirical studies do not find a significant and positive relation between the level of human capital, income level or growth rate. It seems that there are two main reasons for the lack of association between human capital and income mentioned above. The first one

1 Os autores gostariam de agradecer os comentários de Márcio Salvato, Gabriel Porcile, Maurício Bittencourt, Fábio Gomes, Marcos Flávio da Cunha Resende e dos pareceristas anônimos da Revista de Economia.

2 Doutor em Economia pelo Cedeplar/UFMG e professor adjunto da UFPR. E-mail: luciano.nakabashi@ufpr.br.

3 Doutora em Economia pela University of Nottinghan e professora adjunta do Cedeplar/UFMG. E-mail: lizia@ cedeplar.ufmg.br.
} 
is that the relationship between them seems to be more complex than suggested by some endogenous growth models, as the Lucas (1988) and the Uzawa (1965) type. The second is the human capital proxy that is generally used in empirical studies. The present study's goal is to deal with the second problem by means of a human capital proxy that incorporates quantitative and qualitative characteristics of this factor of production. The empirical study and data are based on Mankiw, Romer and Weil's 1992 study and it will be used as a standard to be compared with our results.

Key words: Human capital; empirical results; new proxy.

JEL: C21; E10; I2O; O11; O41; O50

\section{Introdução}

O capital humano é tido como um fator importante sobre o crescimento, pois, como enfatizado por Lucas (1988) e Uzawa (1965) ${ }^{4}$, ele afeta diretamente as habilidades do trabalhador. Um trabalhador mais preparado pode fazer o mesmo serviço, utilizando as mesmas técnicas, máquinas e equipamentos, obtendo um produto final maior do que outro que não esteja tão bem preparado. O capital humano também afeta indiretamente a produção, dadas as externalidades por ele geradas, que atenuam os efeitos dos rendimentos decrescentes do capital, como presente no modelo de Lucas (1988). Além disso, trabalhadores mais qualificados aceleram o processo de difusão, questão crucial para os países em desenvolvimento, como salientado por Nelson e Phelps (1966) e Barro e Sala-iMartin (1997). Uma quarta via é a da capacitação dos indivíduos que estão engajados nos processos de $\mathrm{P} \& \mathrm{D}$ ou qualquer outro processo que afete a criação de tecnologia. Assim P\&D depende da quantidade de capital humano das pessoas empregadas nesse processo, como ressaltado por Romer (1990a).

O objetivo desse trabalho é o de se utilizar uma proxy para capital humano que leve em conta aspectos quantitativos e também qualitativos para se medir com um maior nível de precisão os impactos diretos desse fator sobre o diferencial do nível de renda entre os países. Isso é importante, pois, ao se utilizar uma proxy para capital humano que leve em conta apenas aspectos quantitativos, esta acaba se distanciando muito do estoque de capital humano existente na realidade. $O$ diferencial de qualidade no sistema de formação desse fator entre os diversos países é muito grande para ser ignorado na análise empírica. O estudo empírico será feito através de uma análise comparativa com o modelo de Solow estendido, conforme apresentado no trabalho de Mankiw, Romer e Weil (1992). Assim, o foco principal está apenas na análise dos efeitos diretos 5 .

4 Ou Lucas-Uzawa.

5 Por efeitos/impactos diretos nós nos referimos à introdução do capital humano na função de produção como um dos fatores de produção. Essa forma de incorporação do capital humano busca capturar os efeitos desse fator sobre o nível de renda através da melhora das habilidades dos trabalhadores em suas respectivas funções. 
O método proposto de mensuração das proxies para capital humano que leve em conta aspectos qualitativos e quantitativos consiste em multiplicar a proxy de Mankiw, Romer \& Weil (1992) ${ }^{6}$ pelo Índice de Desenvolvimento Humano7 (IDH) e pelo IDH ao quadrado. A suposição por trás da utilização dessa proxy é de que a qualidade do sistema educacional depende do nível de desenvolvimento do país em questão, sendo o IDH a variável utilizada para tal mensuração. Assim, quanto mais desenvolvido for o país, melhor o seu sistema educacional e a qualidade do capital humano gerada. Por exemplo, ao considerar dois países com a mesma fração da população matriculada no ensino secundário (proxy meramente quantitativa utilizada por MRW), consideramos que o país com maior nível de IDH será aquele com maior quantidade de capital humano, pois o seu sistema formador de capital humano é melhor. O emprego IDH e IDH ao quadrado é para permitir a possibilidade de uma relação não linear.

O emprego do IDH se deve por este ser um indicador bem estabelecido na literatura sobre desenvolvimento econômico, além dos dados estarem disponíveis para uma ampla gama de países e por eles serem de razoável confiabilidade. Portanto, nosso objetivo é mensurar o impacto dessas proxies sobre o nível ${ }^{8}$ e sobre a taxa ${ }^{9}$ de crescimento da renda per capita do grupo de países utilizado por Mankiw, Romer \& Weil (1992, doravante MRW), no mesmo período (1960-1985), fazendo uma análise comparativa com o trabalho desses autores. ${ }^{10}$

Além dessa introdução, o artigo faz uma breve revisão da literatura empírica sobre o capital humano, na seção 2. Na seção 3 descreve-se o modelo; na seção 4 está exposta a metodologia e os dados empregados, enquanto na seção 5 se encontra a análise empírica do impacto do capital humano sobre o nível e a taxa de crescimento da renda, testando a robustez da nova variável ao comparar com os resultados do trabalho de MRW.

\section{Importância empírica do capital humano no crescimento}

Em períodos anteriores ao século XIX, já existiam evidências que apontavam para a importância do investimento em capital humano no processo de desenvolvimento. Easterlin (1981) argumenta que os países da Europa Ocidental que tinham maior nível educacional foram os primeiros a se de-

\footnotetext{
6 Fração da população matriculada no ensino secundário multiplicada pela população em idade de estar no ensino secundário dividida pela população economicamente ativa (UNESCO, Statistical Yearbook).

7 O IDH é um índice composto pela expectativa de vida ao nascer, pela taxa de alfabetização das pessoas acima de 15 anos, por um composto das taxas de matrícula do ensino primário, secundário e terciário e pela renda per capita corrigida pela paridade do poder de compra.

8 Quando se considera que os países estão no estado estacionário.

9 Quando se considera a possibilidade de convergência condicional.

10 Por ser apenas um trabalho comparativo, não controlamos para a questão de causalidade, pois o mesmo problema não foi considerado no estudo de MRW.
} 
senvolverem. Adicionalmente, os países do sudeste e do leste da Europa só conseguiram alcançar um bom nível de desenvolvimento após atingirem um determinado nível educacional. Fora da Europa, os países que tiveram sucesso no processo de desenvolvimento foram os mesmos que alcançaram bons níveis educacionais, como Estados Unidos, Argentina e Japão.

Krueger (1968) faz uma análise sobre o diferencial do nível de renda dos Estados Unidos em relação a alguns países subdesenvolvidos, no intuito de verificar quais os fatores relevantes na explicação da disparidade de renda existente entre eles, com a conclusão de que as proxies $^{11}$ para capital humano explicam mais de $50 \%$ da mesma.

Barro (1991) utiliza várias regressões para analisar o papel do capital humano no processo de crescimento. Os resultados encontrados não rejeitam a hipótese de que o capital humano, medido pelas taxas de matrícula nos ensinos primário e secundário (1960, início do período analisado), afeta positiva e significativamente a taxa de crescimento da renda per capita (1960-1985). Também para o período 1960-1985, outro estudo que mostra que o capital humano é importante na explicação da diferença de renda per capita entre os países é o realizado por MRW, utilizando o modelo ampliado de Solow.

Barro (2000), usando dados de Barro e Lee (2001) para capital humano ${ }^{12}$ e controlando para variáveis como renda inicial, consumo do governo, medida de abertura comercial, taxa de inflação, taxa de fecundidade, termos de troca e um índice para medir o grau de aplicabilidade da lei de propriedade privada, encontra uma relação positiva e significativa entre ensino secundário e de nível superior, para homens com 25 anos ou mais, no início do período, e taxa de crescimento da renda per capita.

Não se pode, porém, tirar conclusões de que o crescimento é uma conseqüência direta do processo de educação da força de trabalho. Resultados adversos seja em relação a direção de causalidade, presença de externalidades, sinal e significância do coeficiente - foram encontrados em inúmeros trabalhos, levando a uma certa incredulidade na forma como o capital humano afeta o crescimento da renda.

Entre os resultados adversos estão vários estudos que apontam à possibilidade de uma relação inversa de causalidade: “... diversos autores manifestaram um certo desconforto em relação à direção da causalidade no relacionamento entre escolaridade e crescimento, sendo a possibilidade de causalidade reversa explicitamente considerada." (Bonelli 2002:842) Bils e Klenow (2000) analisam o impacto do capital humano sobre a renda per capita ${ }^{13}$, controlando para o

11 Krueger utilizou três proxies para medir a diferença de capital humano entre os países: a faixa etária das pessoas que estão cursando o primeiro e segundo graus, além da graduação; anos de escola; e a estrutura setorial da economia, que é a distribuição da população entre residentes urbanos e do campo.

12 Ele utiliza o PIB per capita e, posteriormente, a taxa de investimento em relação ao PIB como variáveis dependentes para a média de três períodos: 1965-75, 1975-85 e 1985-95 (1985-92 para investimento).

13 O período de análise vai de 1960 a 1990. 
fato de que expectativas de maior crescimento futuro da renda possam afetar investimentos em capital humano no presente, concluindo que as diferenças nas taxas de crescimento do capital humano explicam uma fração relativamente pequena das variações nas taxas de crescimento econômico entre os países. Em um estudo para uma série de países, no período de 1960 a 1985 , Romer (1990b), através do uso de variáveis instrumentais, não encontra um coeficiente significativo para a variável capital humano na explicação do crescimento da renda.

Em outros estudos não se confirma a existência de uma correlação entre crescimento e aumento do nível educacional ou se encontra até mesmo uma correlação negativa, como no estudo de Pritchett (2001).

Benhabib e Spiegel (1994) fazem uma série de regressões para testar a importância do capital humano na renda per capita. Os resultados encontrados são diferentes daqueles esperados pela teoria do capital humano. Nos primeiros resultados, os autores chegaram à conclusão de que o impacto do crescimento do capital humano sobre o crescimento da renda per capita não é significativo e o sinal do coeficiente de regressão é negativo. Esse resultado é robusto para mudanças na especificação do modelo e para diferentes fontes de dados. Resultados semelhantes são encontrados por Islam (1995), utilizando a base de dados montada por Summer e Heston (1988) e a de Barro e Lee (1993) para capital humano, por De Gregorio (1992), em um trabalho com doze países da América Latina, no período 1950-1980, com o emprego do método de dados de painel, e por Hall e Jones (1998), em uma análise que emprega o método da contabilidade do crescimento.

O debate empírico não é, portanto, conclusivo com relação à importância do capital humano sobre o crescimento, havendo necessidade de avanço no mesmo. Mesmo em estudos que encontram um papel não significativo do capital humano na renda (taxa ou crescimento), há reservas em relação aos resultados.

Temple (1999) acredita na existência de erros de especificação nos estudos macroeconômicos. Adicionalmente, evidências no nível micro sugerem que aumentos salariais acompanhando a escolaridade adicional são universais, o que contribui para a noção de que aumentos na escolaridade causam crescimento da produtividade e elevam, desse modo, o crescimento da renda (Dowrick 2003). Outro problema é que as proxies para capital humano, em geral, não levam em consideração a capacitação dos indivíduos no trabalho e a diferença de qualidade no ensino dos vários países.

De maneira geral, nos estudos onde não se encontra uma relação significativa ou se encontra, mas com o sinal contrário ao esperado, é fornecida alguma explicação para o fenômeno, de modo a preservar a possível importância da relação entre elas. As explicações geralmente vão em duas direções. A pri- 
meira é a discrepância existente entre a variável capital humano empregada nos modelos teóricos e a proxy geralmente utilizada em estudos empíricos, principalmente em relação ao aspecto qualidade do capital humano. A segunda é a especificação da função de produção, que simplifica em demasia a relação entre essa variável e a renda (Islam 1995).

Esses dois pontos também são enfatizados por Sachs e Warner (1997), que afirmam que, em estudos que utilizam uma comparação entre diversos países, a proxy para capital humano precisa ser melhorada, além de que a base teórica utilizada para explicar a acumulação de capital humano está muito aquém do processo que ocorre no mundo real, especialmente para países com baixo nível de desenvolvimento.

Considerar a possibilidade de dupla causalidade também pode ser importante na análise do capital humano sobre o crescimento. Em um estudo para o período 1960-1990 com intervalo de cinco anos e utilizando a mesma base de dados de MRW e Islam (1995), Freire-Serén (2001) chega à conclusão de que o capital humano é um fator que afeta o nível de renda de forma direta mesmo quando se considera o papel do crescimento da renda sobre o capital humano utilizando o método dos mínimos quadrados não-linear em dois estágios. Os resultados mudam significativamente quando se introduz a variável instrumental na análise (valores preditos da proxy para capital humano), com o coeficiente da proxy para capital humano aumentando de modo considerável e passando a ser significativo.

Apesar de serem minoria, alguns estudos tentam analisar o impacto da educação sobre o crescimento controlando para a sua qualidade. Um exemplo é o estudo de Hanushek e Kimko (2000). Os resultados encontrados indicam uma forte relação entre qualidade da educação e crescimento da renda per capita. Barro (2000), utiliza o TIMMS14 para estudantes e o IALS15 para adultos como proxies para qualidade do capital humano. Ele encontra uma relação positiva entre os testes e as taxas de crescimento da renda real per capita. Utilizando como proxy para qualidade do capital humano, os gastos reais anuais em educação para 48 estados americanos separados em cinco regiões, para os anos de 1880, 1900, 1920 e 1950, Connolly (2004) encontra resultados semelhantes.

Cohen e Soto (2001) utilizaram a equação de Mincer (1974) para construir uma proxy que refletisse melhor o estoque de capital humano do que variáveis como anos de escola. A utilização dessa variável no modelo de Solow estendido melhora os resultados de forma considerável. Para o Brasil, Carpena e Oliveira (2002) realizaram uma estimativa do estoque do capital humano para o período 1981-1999 utilizando métodos semelhantes ao de Cohen e Soto (2002). As conclusões do trabalho são de que a elevação do estoque de capital

14 The Third International Mathematics and Science Study in 1994 and 1995.

15 International Adult Literacy Survey. 
humano per capita variou entre $1 \%$ a $3 \%$ ao ano, gerando um crescimento de 40\% no período. No entanto, a sua evolução é bem próxima da exibida pelo nível médio de escolaridade.

A literatura empírica sobre capital humano tem, em função dessas considerações, procurado: a) estabelecer diferentes especificações para os modelos econométricos, em particular, buscando explicitar os efeitos indiretos do capital humano; b) questionar as formas de estimação dos modelos, causalidades e problemas amostrais; e c) aprimorar as proxies para o capital humano. $\mathrm{O}$ presente trabalho foca neste último item.

\section{O modelo}

\subsection{Estado estacionário}

MRW incluem a variável capital humano de maneira explícita no modelo formal, que eles chamam de modelo ampliado de Solow, além de considerarem as distintas taxas de crescimento da força de trabalho entre os países. Diferentemente do modelo de Lucas-Uzawa, a inclusão do capital humano não leva a um aumento da taxa de crescimento de longo prazo, assim não gera crescimento endógeno. Esse resultado é decorrente da hipótese de que a soma de ambos os capitais não é o suficiente para trazer crescimento sustentado e que a soma deles com o fator trabalho trazem retornos constantes de escala, de acordo com a equação abaixo:

$$
Y_{t}=K_{t}^{\beta} H_{t}^{\alpha}\left(A_{t} L_{t}\right)^{1-\alpha-\beta}
$$

Em que $K_{t}, H_{t}$ e $L_{t}$ são as respectivas quantidades de capital físico, humano e de trabalho no período $t$, e $\alpha, \beta$, e $1-\alpha-\beta$ são as participações dos fatores capital humano, físico e trabalho na renda, respectivamente. Dividindo ambos os lados da equação (1) pela quantidade efetiva de trabalho (AL), chega-se a

$$
\hat{y}=\hat{k}^{\beta} \hat{h}^{\alpha}
$$

Em que $\hat{y}=Y / A L, \hat{k}=K / A L$ e $\hat{h}=H / A L$ são as quantidades por unidades efetivas de trabalho. Com as mesmas suposições do modelo de Solow (1956), mas utilizando a função de produção dada pela equação (1), e utilizando sk e sh para denominar a fração da renda investida em capital físico e humano, respectivamente, as evoluções da acumulação dos capitais físico e humano são determinadas por:

$$
\hat{k}=s_{k} \hat{y}-(\delta+n+g) \hat{k} ; \quad \hat{h}=s_{h} \hat{y}-(\delta+n+g) \hat{h}
$$


Em que $n$ é a taxa de crescimento populacional, g a taxa de progresso tecnológico e $\delta$ a taxa de depreciação do capital. No estado estacionário, as equações em (3) se igualam a zero, formando um sistema de duas equações e duas variáveis endógenas: as quantidades de capital humano e físico, por unidades efetivas de trabalho. Resolvendo para essas variáveis, temos:

$$
\hat{k}^{*}=\left(\frac{s_{k}^{1-\alpha} s_{h}^{\alpha}}{\delta+n+g}\right)^{1 / 1-\alpha-\beta} ; \quad \hat{h}^{*}=\left(\frac{s_{k}^{\beta} s_{h}^{1-\beta}}{\delta+n+g}\right)^{1 / 1-\alpha-\beta}
$$

Em que o sobrescrito * denota que a variável se encontra no estado estacionário. Substituindo as equações em (4) na equação (2), lembrando que $A_{t}=A_{O} e^{g t}$ e transformando ambos os lados da equação em $l n$, chega-se a:

$\ln y^{*}=\ln A(0)+g t+\left(\frac{\beta}{1-\alpha-\beta}\right) \ln \left(s_{k}\right)+\left(\frac{\alpha}{1-\alpha-\beta}\right) \ln \left(s_{h}\right)-\left(\frac{\alpha+\beta}{1-\alpha-\beta}\right) \ln (\delta+n+g)$

Em que $y^{*}$ é a renda per capita no estado estacionário. É feita a suposição de que $g$ e $\delta$ são constantes entre os países. Como o termo $A(o)$ representa não somente tecnologia, mas também dotação de recursos, clima, instituições e outras variáveis, ele deve variar entre os países. Assim, MRW assumem que:

$$
\operatorname{Ln} A(0)=a+\varepsilon
$$

Em que a é uma constante e $\mathcal{E}$ representa a especificidade de cada país. Substituindo essa equação em (5), chega-se a:

$$
\ln \left(y^{*}\right)=a+g t+\left(\frac{\beta}{1-\alpha-\beta}\right) \ln \left(s_{k}\right)+\left(\frac{\alpha}{1-\alpha-\beta}\right) \ln \left(s_{h}\right)-\left(\frac{\alpha+\beta}{1-\alpha-\beta}\right) \ln (\delta+n+g)+\varepsilon
$$

A equação (7) é utilizada na análise de regressão do trabalho de MRW e será utilizada para testarmos a nova proxy para capital humano.

\subsection{Convergência}

Como não há nenhuma garantia de que os países estejam no estado estacionário, é importante que se inclua a possibilidade de convergência, ou seja, de que eles estejam em um período de transição. Próximo ao estado estacionário, a velocidade de convergência é dada por:

$$
\frac{d \ln \left(\hat{y}_{t}\right)}{d t}=\lambda\left[\ln \left(\hat{y}^{*}\right)-\ln \left(\begin{array}{l}
\hat{y}_{t} \\
)
\end{array}\right]\right.
$$


Em que $\lambda=(n+g+\delta)(1-\alpha-\beta) \hat{y}^{*}$ e é a renda em unidades efetivas de trabalho no estado estacionário. A equação (8) implica em:

$$
\ln \left(\hat{y}_{t}\right)=\left(1-e^{-\lambda t}\right) \ln \left(\hat{y}^{*}\right)+e^{-\lambda t} \ln \left(\hat{y}_{0}\right)
$$

Em que $\hat{y}_{0}$ é a renda em unidades efetivas de trabalho, em um dado período inicial. Subtraindo $\ln \left(\hat{y}_{0}\right)$ de ambos os lados da equação (9) e substituindo $\left(\begin{array}{l}\wedge^{*} \\ y\end{array}\right)$, que pode ser derivado da equação (7), chega-se a:

$$
\begin{aligned}
& \ln \left(\hat{y}_{t}\right)-\ln \left(\hat{y}_{0}\right)=\left(1-e^{-\lambda t}\right)\left(\frac{\beta}{1-\alpha-\beta}\right) \ln \left(s_{k}\right)+\left(1-e^{-\lambda t}\right)\left(\frac{\alpha}{1-\alpha-\beta}\right) \ln \left(s_{h}\right) \\
& -\left(1-e^{-\lambda t}\right)\left(\frac{\alpha+\beta}{1-\alpha-\beta}\right) \ln (n+g+\delta)-\left(1-e^{-\lambda t}\right) \ln \left(\hat{y}_{0}\right)+\left(1-e^{-\lambda t}\right) \varepsilon
\end{aligned}
$$

O coeficiente do $\ln \left(\hat{y}_{0}\right)$ do lado direito da equação (10) testa a hipótese de que os países estão fora do estado estacionário e de que há convergência.

\section{Dados e metodologia}

A análise feita abrange o mesmo período do estudo de MRW (1960-1985) e os dados para capital físico e humano, PIB por adulto nos anos de 1960 e 1985 e a taxa de crescimento da população em idade de trabalhar foram retirados do artigo acima mencionado e estão disponíveis no apêndice do mesmo. ${ }^{16} \mathrm{~A}$ variável que serve de proxy para capital humano é a porcentagem da população em idade de trabalhar que está matriculada no ensino secundário. Os dados de MRW ${ }^{17}$ estão divididos em três amostras: a primeira para os países não produtores de petróleo cujos dados estavam disponíveis $(n=98)$; a segunda amostra retira os países em que os dados receberam nota D por Summer e Heston e com uma população abaixo de 1 milhão de habitantes; e a terceira é composta pelos países da OCDE.

Para as seguintes variáveis, MRW utilizam o valor médio do período de estudo: crescimento da população em idade de trabalhar; investimentos como proporção da renda; e a proxy para capital humano (School). Iremos 16 Os dados, por sua vez, são provenientes de base de dados de Summers e Heston (1988), do Banco Mundial (World Tables e World Development Report 1988), e os dados para capital humano são da UNESCO (Statistical Yearbook de vários anos). 
transformar a proxy de capital humano de MRW através do uso do IDH. O IDH é o Índice de Desenvolvimento Humano das Nações Unidas (Human Development Report 2003), estando disponível para o período 1975-1985, em intervalos de cinco anos. O valor deste também é calculado como a média do período. No entanto, como o período é menor, o IDH utilizado é composto pela média aritmética dos anos 1975, 1980 e 1985. Para os países que não possuem os valores do IDH para esses anos, mas os têm para outros, os valores foram calculados de acordo com a média de crescimento do IDH dos países para os quais os dados estão disponíveis. Na amostra equivalente à maior de MRW não constam Somália e Libéria por eles não possuírem dados do IDH. Assim, a maior amostra é composta por 96 países, enquanto que as demais são constituídas pelos mesmos grupos de países do trabalho de MRW.

Pela composição da variável utilizada como proxy para capital humano no presente estudo, seria de se esperar alguns problemas básicos, como um maior nível de correlação entre ela e a variável dependente (no modelo econométrico a ser testado exposto na equação (7)) pelo simples fato desta estar incluída na construção do IDH. Mas a relação deixa de ser espúria se, de fato, os países com maior PIB per capita são os mais desenvolvidos. Assim, a parcela do IDH formada pelo PIB per capita estaria capturando esse maior desenvolvimento. Adicionalmente, proxies para capital humano que possuem aspectos meramente quantitativos trazem erros que podem acarretar em grandes alterações dos resultados. Pela tabela a seguir, com valores da variável School de MRW, vemos que cada uma das colunas é formada por grupos que supostamente teriam semelhante nível de capital humano.

\section{TABELA 1 - VARIÁVEL SCHOOL DE MRW PARA PAÍSES SELECIONADOS}

\begin{tabular}{cccccc}
\hline GRUPO 1 & SCHOOL & GRUPO 2 & SCHOOL & GRUPO 3 & SCHOOL \\
\hline Argélia & 4,5 & Egito & 7,0 & Bahrain & 12,1 \\
Colômbia & 6,1 & Equador & 7,2 & Barbados & 12,1 \\
Zimbábue & 4,4 & Peru & 8,0 & Filipinas & 10,6 \\
Gana & 4,7 & Síria & 8,8 & Guiana & 11,7 \\
Argentina & 5,0 & Sri Lanka & 8,3 & Jamaica & 11,2 \\
Portugal & 5,8 & Venezuela & 7,0 & Panamá & 11,6 \\
Suíça & 4,8 & Alemanha & 8,4 & Austrália & 9,8 \\
& & Áustria & 8,0 & Canadá & 10,6 \\
& & Espanha & 8,0 & Dinamarca & 10,7 \\
& & França & 8,9 & Estados & 10,9 \\
& & Itália & 7,1 & Holanidos & 10,7 \\
\hline
\end{tabular}

FONTE: Elaboração própria a partir dos dados de Mankiw, Romer e Weil (1992)

17 "We begin with data on the fraction of the eligible population (aged 12 to 17) enrolled in secondary school... We then multiply this enrollment rate by the fraction of working-age population that is of school age (aged 15 to 19$). "$ 
Em relação à especificação da equação (10), cabe lembrar que a possibilidade da existência de correlação espúria pelo fato da renda per capita fazer parte do IDH é remota, pois o IDH utilizado é uma média composta por períodos anteriores em relação à variável dependente e, caso se tenha convergência condicional, seria de se esperar o oposto.

A não utilização de variáveis intrumentais para o controle de bicausalidade entre renda per capita e capital humano é por se estar utilizando um período defasado para a proxy da variável capital humano, minimizando a chance dos resultados estarem captando a causalidade reversa, e também para se ter uma comparação direta com os resultados de MRW.

\section{Resultados}

Todas as variáveis estão em logaritmo natural (ln), nas tabelas a serem apresentadas no decorrer do trabalho. A Tabela 2 mostra os resultados de MRW (teste do modelo econométrico exposto em (7)). Eles são apresentados para fins de comparação com os resultados obtidos empregando as novas proxies para capital humano: scidh para o ln de school vezes IDH; e scidh2 para o ln de school vezes IDH ao quadrado.

Nas três primeiras colunas estão os resultados de MRW sem consideração da variável capital humano. A diferença entre as regressões é o tamanho da amostra (N). As três últimas levam em conta capital humano. Na primeira coluna estão as variáveis explicativas, onde I/GDP denota a quantidade de investimentos dividida pelo PIB, $n+g+\delta$ representa a depreciação efetiva do capital físico, $\operatorname{com} n$ sendo a taxa de crescimento populacional, $g$ a taxa de progresso tecnológico, $\delta$ a taxa de depreciação do capital e school é a proxy de MRW para capital humano.

\section{TABELA 2 - MODELO DE SOLOW E DE SOLOW AMPLIADO - RESULTADOS DE MRW}

\begin{tabular}{ccccccc}
\hline \multicolumn{7}{c}{ VARIÁVEL DEPENDENTE: LN DA RENDA PER CAPITA 1985 } \\
\hline \multirow{2}{*}{$\mathrm{I} / \mathrm{GDP}$} & $(1)$ & $(2)$ & $(3)$ & $(4)$ & $(5)$ & $(6)$ \\
& 1,424 & 1,318 & 0,500 & 0,697 & 0,700 & 0,276 \\
$\mathrm{n}+\mathrm{g}+\delta$ & $(9,95)^{* *}$ & $(7,71)^{* *}$ & $(1,15)$ & $(5,25)^{* *}$ & $(4,65)^{* *}$ & $(0,71)$ \\
& $-1,990$ & $-2,017$ & $-0,742$ & $-1,745$ & $-1,500$ & $-1,076$ \\
& $(3,53)^{* *}$ & $(3,38)^{* *}$ & $(0,87)$ & $(4,20)^{* *}$ & $(3,72)^{* *}$ & $(1,42)$ \\
school & & & & 0,654 & 0,731 & 0,768 \\
& & & & $(9,00)^{* *}$ & $(7,67)^{* *}$ & $(2,62)^{* *}$ \\
cons. & $-1,128$ & $-0,722$ & 5,719 & 0,622 & 1,202 & 3,830 \\
& $(0,79)$ & $(0,55)$ & $(2,13)^{*}$ & $(0,58)$ & $(1,18)$ & $(1,56)$ \\
$\mathrm{N}$ & 98 & 75 & 22 & 98 & 75 & 22 \\
R2 & 0,60 & 0,60 & 0,11 & 0,79 & 0,78 & 0,35 \\
R2a. & 0,59 & 0,59 & 0,01 & 0,78 & 0,77 & 0,24 \\
\hline
\end{tabular}

NOTAS: Valores absolutos das estatísticas $t$ estão entre parênteses: * significativo ao nível de $5 \%$; * significativo ao nível de $1 \%$. I/GDP denota a quantidade de investimentos dividida pelo PIB, $\mathrm{n}+\mathrm{g}+\delta$ representa a depreciação efetiva do capital físico, school é porcentagem da população em idade de trabalhar que está matriculada no ensino secundário, cons. é o intercepto, N é o tamanho da amostra, R2 é o coeficiente de determinação e R2a. é o coeficiente de determinação ajustado. 
A proxy para capital humano entra significativamente nas três amostras. Sua introdução reduz o coeficiente do capital físico, além de melhorar o ajuste da regressão, principalmente para os países da OCDE. De forma geral, a introdução da proxy para capital humano melhora a eficiência do modelo de Solow. A Tabela 3 apresenta os resultados com as variáveis scidh e scidh2. As regressões são equivalentes às três últimas da Tabela 2. Nas três primeiras colunas estão os resultados com scidh, enquanto que nas demais, com scidh2.

\begin{tabular}{|c|c|c|c|c|c|c|}
\hline \multicolumn{7}{|c|}{ VARIÁVEL DEPENDENTE: LN DA RENDA PER CAPITA 1985} \\
\hline \multirow{3}{*}{ I/GDP } & (1) & (2) & (3) & (4) & (5) & (6) \\
\hline & 0,578 & 0,519 & 0,189 & 0,495 & 0,424 & 0,130 \\
\hline & $(4,53)^{* *}$ & $(3,58)^{* *}$ & $(0,52)$ & $(3,99)^{* *}$ & $(2,98)^{* *}$ & $(0,38)$ \\
\hline \multirow[t]{2}{*}{$\mathrm{n}+\mathrm{g}+\delta$} & $-1,463$ & $-1,258$ & $-0,841$ & $-1,348$ & $-1,119$ & $-0,613$ \\
\hline & $(3,85)^{* *}$ & $(3,37)^{* * *}$ & $(1,22)$ & $(3,73)^{* *}$ & $(3,13)^{* *}$ & $(0,95)$ \\
\hline \multirow[t]{2}{*}{ scidh } & 0,532 & 0,614 & 0,775 & & & \\
\hline & $(10,35)^{* * *}$ & $(9,18)^{* * *}$ & $(3,34)^{* * *}$ & & & \\
\hline \multirow[t]{2}{*}{ scidh2 } & & & & 0,443 & 0,509 & 0,723 \\
\hline & & & & $(11,32)^{* *}$ & $(10,00)^{* *}$ & $(3,88)^{* * *}$ \\
\hline \multirow[t]{2}{*}{ cons. } & 2,191 & 2,841 & 4,886 & 3,051 & 3,844 & 5,947 \\
\hline & $(2,20)^{*}$ & $(2,89)^{* *}$ & $(2,24)^{*}$ & $(3,16)^{* *}$ & $(3,95)^{* *}$ & $(2,92)^{* *}$ \\
\hline $\mathrm{N}$ & 96 & 75 & 22 & 96 & 75 & 22 \\
\hline R2 & 0,82 & 0,82 & 0,45 & 0,84 & 0,83 & 0,51 \\
\hline R2a. & 0,82 & 0,81 & 0,36 & 0,83 & 0,83 & 0,43 \\
\hline
\end{tabular}

NOTAS: Valores absolutos das estatísticas $t$ estão entre parênteses: * significativo ao nível de $5 \%$; ** significativo ao nível de $1 \%$. I/GDP denota a quantidade de investimentos dividida pelo PIB, $\mathrm{n}+\mathrm{g}+\delta$ representa a depreciação efetiva do capital físico, scidh é school vezes IDH, scidh2 é school vezes IDH ao quadrado, cons. é o intercepto,

N é o tamanho da amostra, R2 é o coeficiente de determinação e R2a. é o coeficiente de determinação ajustado.

Comparando os resultados da Tabela 3 com os de MRW, é interessante notar que todos os coeficientes diminuem, inclusive o do capital humano, com exceção da regressão 3. Ainda assim, a estatística t se eleva para a variável capital humano, em todos os casos, e a proxy para capital humano passa a ser significativa ao nível de $1 \%$, em todas as regressões. O efeito do capital físico sobre a renda per capita se reduz ainda mais e há uma melhora no ajuste da regressão, principalmente para os países da OCDE. ${ }^{18}$ A alteração na proxy para capital humano tem, portanto, um efeito similar ao da introdução da variável school no trabalho de MRW. Os resultados da utilização de scidh2 são parecidos, só que ainda mais acentuados.

Na Tabela 4 estão os resultados de MRW para o modelo de Solow nas três primeiras colunas e Solow ampliado nas três últimas. Todas as regressões consideram convergência condicional (modelo econométrico da equação (10)) e as variáveis de controle estão do lado esquerdo da referida tabela. A variável dependente passa a ser a diferença do ln da renda em 1985 e ln da renda em 1960, de acordo com a equação (10). Na primeira coluna, além das variáveis anteriores estão Y60, que é o ln da renda per capita, em 1960, e $\lambda$, que é a velocidade de convergência.

18 Por ser um estudo comparativo e estarmos substituindo uma proxy de capital humano por outra tendo, conseqüentemente, o mesmo número de variáveis explicativas, tanto faz utilizar o R2 ou R2 ajustado para verificar se ocorre ou não um melhor ajuste da regressão. 


\section{TABELA 4 - CONVERGÊNCIA CONDICIONAL: MODELO DE SOLOW E SOLOW AMPLIADO - RESULTADOS DE MRW}

\begin{tabular}{lllllll}
\hline \multicolumn{5}{c}{ VARIÁVEL DEPENDENTE: LN DA RENDA DE 1985 MENOS LN DA RENDA DE 1960} \\
\hline \multirow{2}{*}{ Y6o } & $(1)$ & $(2)$ & $(3)$ & $(4)$ & $(5)$ & $(6)$ \\
& $-0,141$ & $-0,228$ & $-0,350$ & $-0,288$ & $-0,366$ & 0,398 \\
\multirow{2}{*}{ I/GDP } & $(2,71)^{* *}$ & $(3,98)^{* *}$ & $(5,32)^{* *}$ & $(4,68)^{* *}$ & $(5,43)^{* *}$ & $(5,67)^{* *}$ \\
& 0,647 & 0,646 & 0,390 & 0,524 & 0,538 & 0,322 \\
& $(7,47)^{* *}$ & $(6,22)^{* *}$ & $(2,21)^{* *}$ & $(6,03)^{* *}$ & $(5,26)^{* *}$ & $(1,91)$ \\
$\mathrm{n}+\mathrm{g}+\delta$ & $-0,302$ & $-0,457$ & $-0,766$ & $-0,506$ & $-0,545$ & $-0,863$ \\
& $(0,99)$ & $(1,49)$ & $(2,22)^{* *}$ & $(1,75)$ & $(1,89)$ & $(2,56)^{*}$ \\
\multirow{2}{*}{ school } & & & & 0,231 & 0,270 & 0,228 \\
& & & & $(3,89)^{* *}$ & $(3,37)^{* *}$ & $(1,57)$ \\
cons. & $-1,061$ & $-0,725$ & 0,344 & $-0,455$ & $-0,012$ & 0,179 \\
& $(1,46)$ & $(1,03)$ & $(0,28)$ & $(0,65)$ & $(0,02)$ & $(0,15)$ \\
$\lambda$ & 0,0061 & 0,0104 & 0,0173 & 0,0137 & 0,0182 & 0,0203 \\
N & 98 & 75 & 22 & 98 & 75 & 22 \\
R2 & 0,40 & 0,38 & 0,68 & 0,49 & 0,47 & 0,72 \\
R2a. & 0,38 & 0,35 & 0,62 & 0,46 & 0,43 & 0,65 \\
\hline
\end{tabular}

NOTAS: Valores absolutos das estatísticas $t$ estão entre parênteses:* significativo ao nível de $5 \%$; ** significativo ao nível de 1\%. Y6o é o ln da renda em 1960, I/GDP denota a quantidade de investimentos dividida pelo PIB, $n+g+\delta$ representa a depreciação efetiva do capital físico, school é porcentagem da população em idade de trabalhar que está matriculada no ensino secundário, cons. é o intercepto, $\lambda$ corresponde ao quanto cada país reduz, em cada ano, o gap da renda per capita entre o período em questão e o estado estacionário (velocidade de convergência), N é o tamanho da amostra, R2 é o coeficiente de determinação e R2a. é o coeficiente de determinação ajustado.

Os resultados indicam a existência de convergência condicional, pois os coeficientes do ln da renda em 1960 (Y60) são negativos e significativos em todas as regressões, além de $\lambda$ ser positivo em todos os casos. A letra $\lambda$ corresponde ao quanto cada país reduz, em cada ano, o gap da renda per capita entre o período em questão e o estado estacionário. Por exemplo, na primeira regressão a redução do gap é de o,6\% ao ano. Comparando as três primeiras regressões com as demais se pode ver que a introdução da proxy para capital humano aumenta a velocidade de convergência, indicando que os países que têm mais capital humano possuem maiores taxas de crescimento no período de transição, ceteris paribus. Além disso, o coeficiente do capital físico se reduz e ocorre uma melhora no ajuste da regressão.

Na Tabela 5 podem ser vistos os resultados das regressões com o emprego das novas proxies para capital humano. Nas três primeiras colunas estão os resultados quando se utiliza a proxy scidh, enquanto que nas três subseqüentes estão os resultados com o emprego da proxy scidh2. 


\section{TABELA 5 - CONVERGÊNCIA CONDICTIONAL: MODELO DE SOLOW AMPLIADO PARA AS NOVAS PROXIES PARA CAPITAL HUMANO}

\begin{tabular}{|c|c|c|c|c|c|c|}
\hline \multicolumn{7}{|c|}{ VARIÁVEL DEPENDENTE: LN DA RENDA DE 1985 MENOS LN DA RENDA DE 1960} \\
\hline & (1) & (2) & (3) & (4) & $(5)$ & (6) \\
\hline \multirow[t]{2}{*}{ Y6o } & $-0,343$ & $-0,414$ & $-0,420$ & $-0,372$ & $-0,442$ & $-0,439$ \\
\hline & $(5,44)^{* *}$ & $(5,89)^{* *}$ & $(5,82)^{* *}$ & $(5,78)^{* *}$ & $(6,16)^{* * *}$ & $(5,93)^{* *}$ \\
\hline \multirow[t]{2}{*}{ I/GDP } & 0,516 & 0,477 & 0,306 & 0,486 & 0,439 & 0,286 \\
\hline & $(5,96)^{* *}$ & $(4,60)^{* * *}$ & $(1,79)$ & $(5,58)^{* *}$ & $(4,18)^{* *}$ & $(1,69)$ \\
\hline \multirow[t]{2}{*}{$\mathrm{n}+\mathrm{g}+\delta$} & $-0,438$ & $-0,519$ & $-0,794$ & $-0,437$ & $-0,494$ & $-0,721$ \\
\hline & $(1,59)$ & $(1,85)$ & $(2,45)^{*}$ & $(1,62)$ & $(1,79)$ & $(2,27)^{*}$ \\
\hline \multirow[t]{2}{*}{ scidh } & 0,201 & 0,254 & 0,239 & & & \\
\hline & $(4,26)^{* * *}$ & $(3,95)^{* * *}$ & $(1,87)$ & & & \\
\hline \multirow[t]{2}{*}{ scidh2 } & & & & 0,179 & 0,224 & 0,233 \\
\hline & & & & $(4,64)^{* *}$ & $(4,27)^{* *}$ & $(2,08)^{* *}$ \\
\hline \multirow[t]{2}{*}{ cons. } & 0,329 & 0,751 & 0,671 & 0,756 & 1,286 & 1,150 \\
\hline & $(0,47)$ & $(1,01)$ & $(0,58)$ & $(1,05)$ & $(1,63)$ & $(0,97)$ \\
\hline$\lambda$ & 0,0168 & 0,0214 & 0,0218 & 0,0186 & 0,0233 & 0,0231 \\
\hline $\mathrm{N}$ & 96 & 75 & 22 & 96 & 75 & 22 \\
\hline $\mathrm{R} 2$ & 0,53 & 0,49 & 0,73 & 0,54 & 0,51 & 0,74 \\
\hline R2a. & 0,51 & 0,46 & 0,67 & 0,52 & 0,48 & 0,68 \\
\hline
\end{tabular}

NOTAS: Valores absolutos das estatísticas $t$ estão entre parênteses:* significativo ao nível de $5 \%$; ** significativo ao nível de $1 \%$. Y6o é o ln da renda em 1960, I/GDP denota a quantidade de investimentos dividida pelo PIB, $n+g+\delta$ representa a depreciação efetiva do capital físico, scidh é school vezes IDH, scidh2 é school vezes IDH ao quadrado, cons. é o intercepto, $\lambda$ corresponde ao quanto cada país reduz, em cada ano, o gap da renda per capita entre o período em questão e o estado estacionário (velocidade de convergência), N é o tamanho da amostra, R2 é o coeficiente de determinação e R2a. é o coeficiente de determinação ajustado.

Um dos efeitos da introdução das novas proxies é o aumento, em valor absoluto, do coeficiente de Y6o e de $\lambda$, indicando uma maior velocidade de convergência, com o efeito para scidh2 sendo mais acentuado. Isso mostra que a qualidade do sistema fomador de capital humano afeta a taxa de crescimento dos países de tal modo que quanto melhor a qualidade, mais rápida é a sua taxa de crescimento, considerando tudo mais constante. Para melhor se entender essa elevação na velocidade de convergência, note que, quando se compara uma ampla gama de países, não há convergência caso não se controle para nenhuma outra variável além do nível inicial da renda per capita, como constatado por MRW. Com a inclusão de outras variáveis de controle, 
podemos verificar a existência de convergência condicional. De modo similar, quando não se leva em conta o fato de que existem aspectos qualitativos no fator capital humano, a velocidade de convergência é menor. ${ }^{19}$

O coeficiente do capital físico diminui em todos os casos, assim como o da depreciação efetiva (em termos absolutos). O coeficiente da proxy para capital humano se reduz nas duas primeiras amostras e se eleva na terceira, além de sua significância aumentar em todos os casos, passando a ser significativo até mesmo no caso dos países da OCDE, quando se faz uso de scidh2. O ajuste da regressão aumenta marginalmente. Em todas as situações os efeitos da utilização de scidh2 vão na mesma direção, mas são mais acentuados.

Com o objetivo de ter maior segurança sobre os resultados das regressões, alguns testes foram realizados. Para medir o grau de multicolinearidade, foi utilizado o fator inflação da variância. Como uma regra prática para mensurar o problema, ele é considerado grave quando o fator inflação da variância é maior do que 10. Isso não acontece em nenhuma das equações acima. ${ }^{20} \mathrm{O}$ valor mais elevado é 3,97 para a variável scidh2 na quarta regressão da Tabela 5 .

Apesar da heterocedasticidade não afetar a propriedade de não viés dos estimadores por MQO, estes já não são eficientes e podem levar a conclusões errôneas quando se faz uso de testes de hipótese. Esse problema também não é sério visto que, ao nível de $1 \%$ de significância, não se rejeita a hipótese nula de que os resíduos são homocedásticos em nenhuma das equações acima. Ao nível de $5 \%$ e mesmo de $10 \%$ só se rejeita a hipótese nula para as amostras intermediárias nos casos em que se considera convergência.

Analisando a assimetria e curtose para testar a normalidade dos resíduos, não se rejeita a hipótese de que eles são normais em nenhuma das regressões, ao nível de $5 \%$. Esses resultados são extremamente importantes visto que todos os testes de hipóteses e, portanto, suas validades dependem desta. Não foi avaliado se existe o problema de autocorrelação dos resíduos por se tratar de dados de corte. ${ }^{21}$

19 Isso acontece porque caso os países que possuem um maior nível de capital humano sejam aqueles que cresçam mais, quando se leva em conta apenas uma proxy que captura aspectos quantitativos pode ocorrer que países com um elevado índice de anos de escola não cresçam muito porque a qualidade do ensino não é boa. Mas quando se controla a qualidade, verifica-se que o nível de capital humano não é tão elevado, o que explica porque a performance do país é relativamente fraca, o que aumenta a velocidade de convergência.

20 Todos os resultados estão disponíveis com os autores.

21 Apesar dos problemas analisados não serem graves, foram feitas regressões com resíduos robustos corrigidos pelo método de White para corrigir para possíveis problemas de heterocedasticidades. Os resultados são muito semelhantes aos apresentados nas tabelas anteriores. Assim, pode-se concluir que caso se tenha heterocedasticidade, esse problema não está alterando os resultados dos testes de forma significativa. 


\section{Conclusões}

A introdução das novas proxies para capital humano foi feita no sentido de se tentar obter resultados empíricos mais confiáveis em estudos que buscam avaliar o papel do capital humano sobre a renda e a taxa de crescimento econômico. O diferencial de qualidade no sistema de formação de capital humano é uma característica marcante quando se considera uma gama tão diversa de países e, portanto, é essencial ser levado em consideração. Além disso, como alertado por Islam (1995), a não consideração do fator qualidade na proxy para capital humano pode levar a resultados em que a conclusão é de que esse fator não é importante de forma direta para o crescimento econômico.

A utilização do Índice de Desenvolvimento Humano (IDH) foi no sentido de mensurar o nível de desenvolvimento dos países. A suposição sobre o efeito do IDH na qualidade do sistema formador de capital humano é a de que países mais desenvolvidos possuem uma melhor infra-estrutura educacional, professores mais preparados, entre outros fatores que façam com que o sistema formador de capital humano seja mais desenvolvido. De fato, o emprego das novas proxies, no presente estudo, trouxe uma melhora no ajuste das regressões. Todos os resultados confirmaram aqueles encontrados por Mankiw, Romer e Weil (MRW), mas o capital humano é um fator ainda mais importante do que se pôde concluir no trabalho dos autores acima mencionados. Ele mostrou ser importante na explicação do diferencial de renda per capita dos países em questão e foi mais significativo do que no estudo de MRW. O capital físico também perde importância com a utilização das novas proxies, possivelmente por ele estar explicando parte do papel que cabe ao fator capital humano no trabalho de MRW.

A elevação da velocidade de convergência também é um indicativo de que a introdução das novas proxies melhora os resultados do modelo, pois é de se esperar que quando se leva em conta diferenças qualitativas existentes na formação desse fator, a velocidade de convergência seja maior. Um exemplo seria dois países em transição, A e B, com semelhantes nível de investimento, taxa de depreciação efetiva de capital e, de acordo com uma medida puramente quantitativa, o mesmo nível de capital humano por trabalhador. Suponha que A possua um nível de renda por trabalhador inicial ligeiramente maior e que ele cresça mais rapidamente. Nesse caso, não há convergência condicional quando se controla pelas variáveis acima listadas. Mas se o capital humano é, realmente, maior no país A e a tal medida para esse fator não captura essa diferença, devido a elementos qualitativos, é provável que se tenha convergência condicional, de fato.

A possibilidade de correlação espúria pela introdução do IDH, que contém a renda per capita na sua construção, é minimizada pelo fato de que esse indicador é usado apenas como ponderador. Sua influência é ainda menor, nas regressões em que os países se encontram fora do estado estacionário, pelo fato do IDH ser uma média composta por períodos anteriores, já que foi confirmada a presença de conver- 
gência condicional e, desse modo, seria de se esperar uma correlação negativa entre o nível da renda per capita em períodos anteriores e a taxa de crescimento desta no último período (1985). Embora seja possível que existam problemas de causalidade entre as variáveis, eles não foram considerados neste trabalho. $\mathrm{O}$ objetivo foi fazer uma comparação com a análise realizada por MRW.

A similaridade dos resultados das regressões com resíduos robustos corrigidos pelo método de White utilizado para remediar problemas de heterocedasticidade confirmam e dão suporte aos resultados encontrados. Concluindo, a utilização das novas proxies torna os resultados mais confiáveis por se ter uma melhor medida de capital humano.

\section{Referências}

BARRO, R. J. (1991). "Economic growth in a cross section of countries." The Quarterly Journal of Economics 106 (2): 407-443.

BARRO, R. J. (2000). Education and economic growth. Cambridge MA: Harvard University, Department of Economics. (Working paper)

BARRO, R. J. \& LEE, J. W. (1993). "International comparisons of educational attainment." Journal of Monetary Economics 32 (3): 363-394.

BARRO, R. J. \& LEE, J. W. (2001). "International data on educational attainment: update and implications." Oxford Economic Papers 53 (3): 541-563.

BARRO, R. J. \& SALA-I-MARTIN, X. (1997). "Technological diffusion, convergence, and growth." Journal of Economic Growth 2 (1): 1-27.

BENHABIB, J. \& SPIEGEL, M. M. (1994). "The role of human capital in economic development: evidence from aggregate cross-country data." Journal of Monetary Economics 34 (2): 143-173.

BILS, M. \& KLENOW, P. J. (2000) "Does schooling cause growth?" The American Economic Review 90 (5): 1160-1182.

BONELLI, R. (2002). "Crescimento, desigualdade e educação: notas para uma resenha com referência ao Brasil." Economia Aplicada 6 (4): 819-873.

CARPENA, L. \& OLIVEIRA, J.B. (2002). "Estimativa do estoque de capital humano para o Brasil: 1981 a 1999.” Texto para Discussão do IPEA (877): 1-21.

COHEN, D. \& SOTO, M. (2001). "Growth and human capital: good data, good results." OECD Development Centre, Technical Papers (179): 1-42.

CONNOLLY, M. P. (2004) "Human capital and growth in the Post-Bellum South: a separate but unequal story." The Journal of Economic History 64 (2): 1-39.

DE GREGORIO, J. (1992) “Economic growth in Latin America." Journal of Developments Economics 39 (1): 59-84.

DOWRICK, S. (2003). Ideas and education: level or growth effects?. Cambridge, MA: National Bureau of Economic Research, p. 30. (Working paper, 9709)

EASTERLIN, R. A. (1981). "Why isn't the whole world developed." The Journal of Economic History 41 (1): 1-21. 
FREIRE-SÉREN, M. J. (2001) "Human capital accumulation and economic growth." Investigaciones Económicas 25 (3): 585-602.

HALL, R. E. \& JONES, C. I. Why some countries produce so much more output per worker than others?. Cambridge, MA: National Bureau of Economic Research, p. 49. (Working paper; 6564)

HANUSHEK, E. A. \& KIMKO, D. D. (2000) "Schooling, labor-force quality, and the growth of nations." The American Economic Review 90 (5): 1184-1208.

HUMAN DEVELOPMENT REPORT (2003). "Millennium development goals: a compact among nations to end human poverty. New York: United Nations Development Program, 2003." URL [On line]: http://hdr.undp.org/reports/global/2003/pdf/hdr03 overview.pdf.

ISLAM, N. (1995). "Growth empirics: a panel data approach." The Quarterly Journal of Economics 110 (4): 1127-1170.

KRUEGER, A. O. (1968). "Factor endowments and per capita income differences among countries.” The Economic Journal 78 (311): 641-659.

LUCAS, R. E. Jr. (1988). "On the mechanics of economic development." Journal of Monetary Economics 22 (1): 3-42.

MANKIW, N. G. \& ROMER, D. \& WEIL, D. (1992). "A contribution to the empirics of economic growth.” The Quarterly Journal of Economics 107 (2): 407-437.

MINCER, J. (1974). Schooling, experience and earnings. Columbia University Press.

NELSON, R. R. \& PHELPS, E. S. (1966). "Investment in humans, technological diffusion, and economic growth." The American Economic Review 56 (2): 69-75.

PRITCHETT, L. (2001). "Where has all the education gone?" The World Bank Economic Review 15 (3): 367-391.

ROMER, P. (1990a). "Endogenous technological change". The Journal of Political Economy, 18 (5): 71-102.

ROMER, P. (1990b). "Human capital and growth: theory and evidence." CarnegieRochester Conference Series on Public Policy (32): 251-286.

SACHS, J. D. \& WARNER, A. M. (1997). "Fundamental sources of long-run growth." The American Economic Review 87 (2): 184-188.

SUMMERS, R. \& HESTON, A. (1988). "A new set of international comparisons of real product and price levels: estimates for 130 countries." Review of Income and Wealth 4 (1): $1-25$.

TEMPLE, J. R. W. (1999). “A positive effect of human capital on growth.” Economic Letters 65 (1): 131-134.

UZAWA, H. (1965). "Optimum technical change in an aggregative model of economic growth.” International Economic Review 6 (1): 18-31.

Submissão: 19 de setembro de 2006

Primeira resposta: 2 de dezembro de 2006

Aceite: 12 de fevereiro de 2007 